\title{
QCD thermodynamics at zero and non-zero density
}

\section{Christian Schmidt*}

Brookhaven National Laboratory

E-mail: cschmidtequark.phy.bnl.gov

\begin{abstract}
We present recent results on thermodynamics of QCD with almost physical light quark masses and a physical strange quark mass value. These calculations have been performed with an improved staggerd action especially designed for finite temperature lattice QCD. In detail we present a calculation of the transition temperature, using a combined chiral and continuum extrapolation. Furthermore we present preliminary results on the interaction measure and energy density at almost realistic quark masses. Finally we disscuss the response of the pressure to a finite quark chemical potential. Within the Taylor expansion formalism we calculate quark number susceptibilities and leading order corrections to finite chemical potential. This is particularly usefull for mapping out the critical region in the QCD phase diagram.
\end{abstract}

The 3rd edition of the International Workshop - The Critical Point and Onset of Deconfinement July 3-7 2006

Galileo Galilei Institute, Florence, Italy

\footnotetext{
* Speaker.
} 


\section{Introduction}

It is by now well established that the properties of matter formed from strongly interacting elementary particles change drastically at high temperatures. Quarks and gluons are no longer confined to move inside hadrons but organize in a new form of strongly interacting matter, the so-called quark-gluon plasma (QGP). The transition from hadronic matter to the QGP as well as properties of the high temperature phase have been studied extensively in lattice calculations over recent years [1]. Nonetheless, detailed quantitative information on the transition and the structure of the high temperature phase in the physical situation of two light and a heavier strange quark $((2+1)$-flavor QCD) is rare [2, 3, 4, 5]. In order to relate experimental observables determined in relativistic heavy ion collisions to lattice results, it is important to achieve good quantitative control, in calculations with physical quark masses, over basic parameters that characterize the transition from the low to the high temperature phase of QCD. The most fundamental quantities characterize bulk properties of hot and dense matter: the transition temperature, energy density and pressure.

Lattice QCD currently is the only quantitative approach to finite temperature QCD based on first principle calculation. At non-zero density however, lattice QCD is harmed by the sign problem ever since its inception. To overcome the sign problem is a challenging and outstanding problem. Nevertheless, during the last few years a lot of progress has been made to circumvent the sign problem for small values of $\mu_{q} / T$, where $\mu_{q}$ is the quark chemical potential and $T$ the temperature $[6,7,8]$

\section{Lattice formulation and calculational setup}

We study the thermodynamics of QCD with two light quarks $\left(\hat{m}_{l} \equiv \hat{m}_{u}=\hat{m}_{d}\right)$ and a heavier strange quark $\left(\hat{m}_{s}\right)$ described by the QCD partition function which is discretized on a four dimensional lattice of size $N_{\sigma}^{3} \times N_{\tau}$,

$$
Z\left(\beta, \hat{m}_{l}, \hat{m}_{s}, N_{\sigma}, N_{\tau}\right)=\int \prod_{x, \mu} \mathrm{d} U_{x, \mu}\left(\operatorname{det} D\left(\hat{m}_{l}\right)\right)^{1 / 2}\left(\operatorname{det} D\left(\hat{m}_{s}\right)\right)^{1 / 4} \mathrm{e}^{-\beta S_{G}(U)} .
$$

Here we will use staggered fermions to discretize the fermionic sector of QCD. The fermions have already been integrated out, which gives rise to the determinants of the staggered fermion matrices, $D\left(\hat{m}_{l}\right)$ and $D\left(\hat{m}_{s}\right)$ for the contributions of two light and one heavy quark degree of freedom, respectively. Moreover, $\beta=6 / g^{2}$ is the gauge coupling constant, $\hat{m}_{s, l}$ denote the dimensionless, bare quark masses in units of the lattice spacing $a$, and $S_{G}$ is the gauge action which is expressed in terms of gauge field matrices $U_{x, \mu} \in S U(3)$ located on the links $(x, \mu) \equiv\left(x_{0}, \mathbf{x}, \mu\right)$ of the four dimensional lattice; $\mu=0, \ldots, 3$.

In our calculations we use a tree level, $\mathscr{O}\left(a^{2}\right)$ improved gauge action, $S_{G}$, which includes the standard Wilson plaquette term and the planar 6-link Wilson loop. In the fermion sector, we use an improved staggered fermion action with 1-link and bended 3-link terms. The coefficient of the bended 3-link term has been fixed by demanding a rotationally invariant quark propagator up to $\mathscr{O}\left(p^{4}\right)$, which improves the quark dispersion relation at $\mathscr{O}\left(a^{2}\right)$. This eliminates $\mathscr{O}\left(a^{2}\right)$ corrections to the pressure at tree level and leads to a strong reduction of cut-off effects in other bulk thermodynamic observables in the infinite temperature limit, as well as in $\mathscr{O}\left(g^{2}\right)$ perturbation theory [9]. 
The 1-link term in the fermion action has been 'smeared' by adding a 3-link staple. This improves the flavor symmetry of the staggered fermion action [10]. We call this action the p4fat 3 action. It has been used previously in studies of QCD thermodynamics on lattices of temporal extent $N_{\tau}=4$ with larger quark masses $[2,11]$.

Our studies of the transition to the high temperature phase of QCD [12] have been performed on lattices of size $N_{\sigma}^{3} \times N_{\tau}$ with $N_{\tau}=4$ and 6 and spatial lattice sizes $N_{\sigma}=8,16,24$ and 32 . We performed calculations for several values of the light to strange quark mass ratio, $\hat{m}_{l} / \hat{m}_{s}$ for fixed $\hat{m}_{s}$. The strange quark mass has been chosen such that the extrapolation to physical light quark mass values yields approximately the correct physical kaon mass value. The range of the light quark mass corresponds to a regime of the pseudo-scalar (pion) mass of $150 \mathrm{MeV} \lesssim m_{p s} \lesssim 500 \mathrm{MeV}$

In order to convert lattice units to physical units, zero temperature calculations are necessary which have been performed on $16^{3} \times 32$ lattices. We use parameters characterizing the shape of the static quark potential $\left(r_{0}, r_{1}, \sqrt{\sigma}\right)$ as well as hadron masses to set the scale for thermodynamic observables.

The numerical simulation of the QCD partition function has been performed using the RHMC algorithm [13]. Unlike the hybrid-R algorithm [14] used in most previous studies of QCD thermodynamics performed with staggered fermions, this algorithm has the advantage of being exact, i.e. finite step size errors arising from the discretization of the molecular dynamics evolution of gauge fields in configuration space are eliminated through an additional Monte Carlo accept/reject step. This is possible with the introduction of a rational function approximation for roots of fermion determinants appearing in Eq. 2.1.

\section{Order parameters and susceptibilities}

To determine the QCD transition temperature and phase diagram, order parameter of the QCD transition are indispensable. In the chiral limit the chiral condensate $\langle\bar{\psi} \psi\rangle$ is the order parameter for the spontaneous chiral symmetry breaking of QCD. On the other hand in the heavy quark limit the Polyakov loop $\langle L\rangle$ is the order parameter of the deconfinement phase transition. For finite quark masses, these observables remain good indicators for the (pseudo) critical point. Especially their susceptibilities are useful to determine the pseudo critical coupling $\beta_{c}$ in numerical simulations.

In Figure 1(left) we compare results for the light quark chiral condensate calculated on lattices of size $8^{3} \times 4$ and $16^{3} \times 4$. It clearly reflects the presence of finite volume effects at small values of the quark mass. While finite volume effects seem to be negligible for $\hat{m}_{l} / \hat{m}_{s} \geq 0.2$, for $\hat{m}_{l} / \hat{m}_{s}=0.1$ we observe a small but statistically significant volume dependence for the chiral condensate as well as for the Polyakov loop expectation value. This volume dependence is even more pronounced for $\hat{m}_{l} / \hat{m}_{s}=0.05$ and seems to be stronger at low temperatures. While the value of the chiral condensate increases with increasing volume the Polyakov loop expectation value decreases (Figure 1(right)).

We use the Polyakov loop susceptibility as well as the disconnected part of the chiral susceptibility to locate the transition temperature to the high temperature phase of QCD,

$$
\begin{aligned}
\chi_{L} & \equiv N_{\sigma}^{3}\left(\left\langle L^{2}\right\rangle-\langle L\rangle^{2}\right), \\
\frac{\chi_{q}}{T^{2}} & \equiv \frac{N_{\tau}}{16 N_{\sigma}^{3}}\left(\left\langle\left(\operatorname{Tr} D^{-1}\left(\hat{m}_{q}\right)\right)^{2}\right\rangle-\left\langle\operatorname{Tr} D^{-1}\left(\hat{m}_{q}\right)\right\rangle^{2}\right), q \equiv l, s .
\end{aligned}
$$



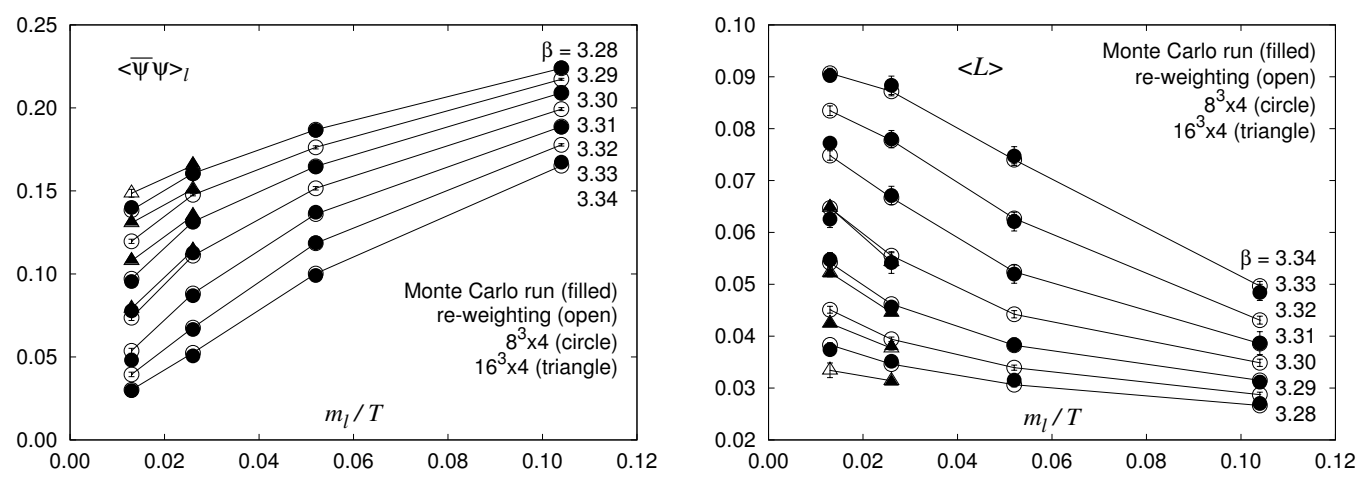

Figure 1: The light quark chiral condensate in units of $a^{-3}$ (left) and the Polyakov loop expectation value (right) as function of the bare light quark mass in units of the temperature, $m_{l} / T \equiv \hat{m}_{l} N_{\tau}$ for fixed $\beta$ and $\hat{m}_{s}=0.065$ on lattices of size $8^{3} \times 4$ (circle) and $16^{3} \times 4$ (triangles). Shown are results for various values of $\beta$ ranging from $\beta=3.28$ to $\beta=3.4$ (top to bottom for $\langle\bar{\psi} \psi\rangle$ and bottom to top for $\langle L\rangle$ ). Full and open symbols show results obtained from direct simulations and Ferrenberg-Swendsen interpolations, respectively.
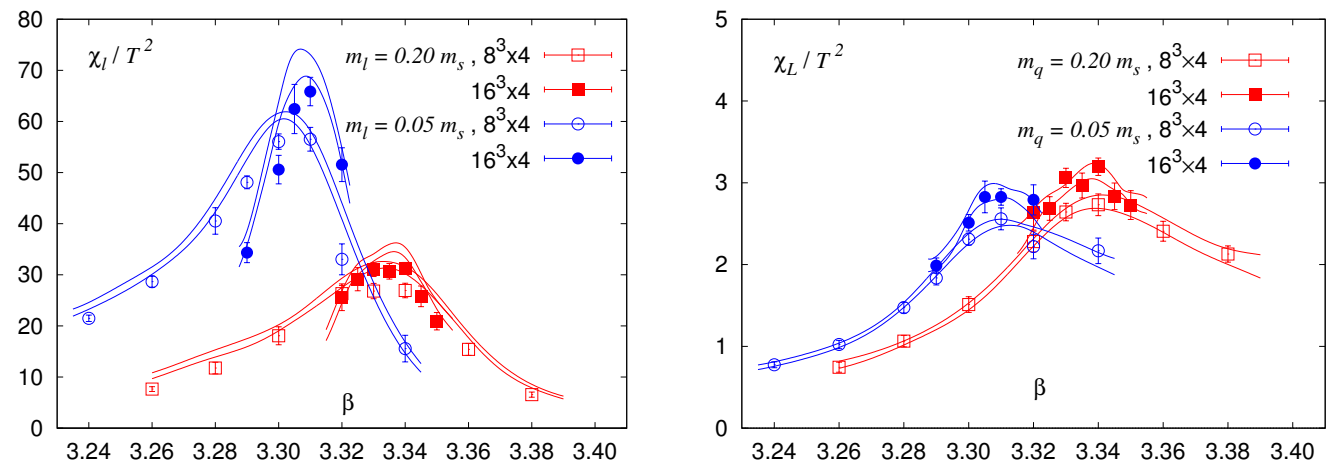

Figure 2: The disconnected part of the light quark chiral susceptibility (left) and the Polyakov loop (right) on lattices of size $8^{3} \times 4$ (squares) and $16^{3} \times 4$ (circles) for two different values of the light quark mass. The curves show Ferrenberg-Swendsen interpolations of the data points obtained from multi-parameter histograms with an error band coming from Ferrenberg-Swendsen reweightings performed on different jackknife samples.

In Figure 2 we show results for the disconnected part of the light quark chiral susceptibility, $\chi_{l}$ and the Polyakov loop, calculated on $8^{3} \times 4$ and $16^{3} \times 4$ lattices. The location of peaks in the susceptibilities has been determined from a Ferrenberg-Swendsen reweighting of data in the vicinity of the peaks. Errors on the critical couplings determined in this way have been obtained from a jackknife analysis where Ferrenberg-Swendsen interpolations have been performed on different sub-samples. In agreement with earlier calculations we find that the position of peaks in $\chi_{l}$ and $\chi_{L}$ show only little volume dependence and that the peak height changes only little, although the maxima become somewhat more pronounced on the larger lattices. This is consistent with the transition being a crossover rather than a true phase transition in the infinite volume limit.

Although differences in the critical coupling extracted from $\chi_{L}$ and $\chi_{l}$ are small we find that on small lattices the peak in the Polyakov loop susceptibility is located at a systematically larger value 
of the gauge coupling $\beta$. In a finite volume this is, of course, not unexpected, and in the infinite volume limit an ambiguity in identifying the transition point may also remain for a crossover transition. Nonetheless, we observe that the difference $\beta_{c, L}-\beta_{c, l}$ decreases with increasing volume and is within errors consistent with zero for $16^{3} \times 4$, which has the largest spatial volume expressed in units of the temperature, $T V^{1 / 3}=4$. On the smallest lattice, $8^{3} \times 4$, we find $\beta_{c, L}-\beta_{c, l} \simeq 0.0077(9)$. Within the statistical accuracy of our data we also do not find any systematic quark mass dependence of this difference, $\beta_{c, L}-\beta_{c, l}$.

In addition to the light quark condensate and its susceptibility we also have analyzed the strange quark condensate and its susceptibility, $\chi_{s}$. We find that the light and heavy quark condensates are strongly correlated, which is easily seen in the MD-time evolution of these quantities. Already on the smallest lattices the position of the peak in the heavy quark susceptibilities is consistent with that deduced from the light quark condensate. On the larger, $N_{\sigma}=16$, lattices the difference $\left|\beta_{c, l}-\beta_{c, s}\right|$ is in all cases zero within statistical errors, which are about $3 \cdot 10^{-3}$. Any temperature difference in the crossover behavior for the light and strange quark sector of QCD, which sometimes is discussed in phenomenological models, thus is below the $1 \mathrm{MeV}$ level.

We also have analyzed derivatives of the QCD partition function with respect to a quark chemical potential (see Section 7). We note here that also from the analysis of the Taylor expansion coefficient $c_{4}\left(d_{4}\right)$ we find values for the pseudo-critical couplings that are in agreement with the above estimates [15] (see the quartic strange quark number fluctuations in Fig. 7 (left)). However, as mentioned above the consistence of pseudo-critical couplings as determined from different observables is not necessary. If the transition becomes smoother closer to the continuum limit different observables may lead to different estimates for the crossover point. In fact, for the stout-link improved staggered action and lattices with temporal extent of $N_{\tau}=8$ to 10, a large difference in $\beta_{c}$ determined from the chiral condensate and the strange quark number fluctuations has been found [16].

\section{Scale setting and the heavy quark potential}

In order to calculate the transition temperature in terms of an observable that is experimentally accessible and can be used to set the scale for $T_{c}$ we have to perform a zero temperature calculation at the critical couplings $\beta_{c}$ determined in the previous section. This will allow us to eliminate the unknown lattice cut-off, $a\left(\beta_{c}\right)$, which determines $T_{c}$ on a lattice with temporal extent $N_{\tau}$, i.e. $T_{c}=1 / N_{\tau} a\left(\beta_{c}\right)$. To do so we have performed calculations at zero temperature, i.e. on lattices of size $16^{3} \times 32$, and calculated several hadron masses as well as the static quark potential. From the latter we determine the string tension and extract short distance scale parameters $r_{0}, r_{1}$, which are defined as separations between the static quark anti-quark sources at which the force between them attains certain values [17],

$$
\left.r^{2} \frac{\mathrm{d} V_{\bar{q} q}(r)}{\mathrm{d} r}\right|_{r=r_{0}}=1.65,\left.r^{2} \frac{\mathrm{d} V_{\bar{q} q}(r)}{\mathrm{d} r}\right|_{r=r_{1}}=1.0
$$

Although these scale parameters are not directly accessible to experiment they can be well estimated from heavy quarkonium phenomenology. Moreover, they have been determined quite accurately in lattice calculations through a combined analysis of the static quark potential [18] and level 


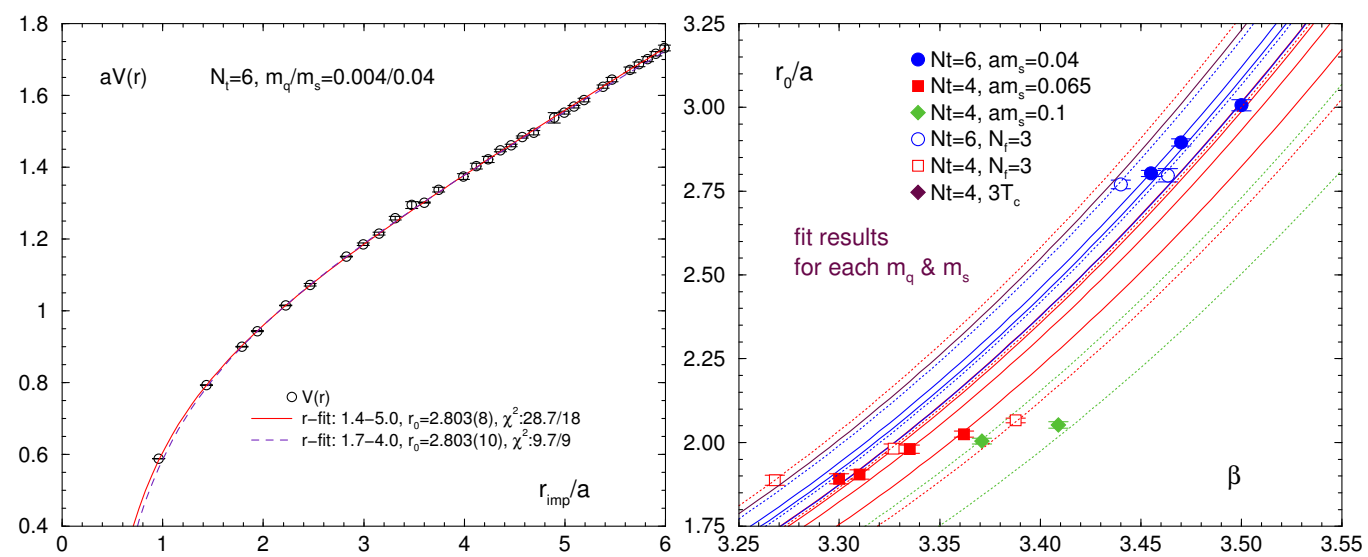

Figure 3: Fit of the static quark potential with the Cornell ansatz and improved distances (left) and fit of the scale parameter $r_{0}$ versus the lattice coupling $\beta$ to a renormalization group inspired ansatz (Eq 4.3) (right).

splittings in bottomonium spectra [19]. Both these calculations have been performed on identical sets of gauge field configurations. We will use the value for $r_{0}$ determined in the bottomonium calculation [19] for all conversions of lattice results to physical units,

$$
r_{0}=0.469(7) \mathrm{fm} .
$$

Our zero temperature calculations have been performed at values of the gauge coupling in the vicinity of the $\beta_{c}$ we found from our analysis of the chiral susceptibilities. We typically generated several thousand configurations and analyzed the hadron spectrum and static quark potential on every $10^{\text {th }}$ configuration. We obtain the scale by using the simple Cornell form to fit our numerical results for the static quark potential, $V_{\bar{q} q}(r)=-\alpha / r+\sigma r+c$. With this fit-ansatz, which does not include a possible running of the coupling $\alpha$, the force entering the definition of $r_{0}$ is easily calculated and we find from Eq. 4.1, $r_{0} \equiv \sqrt{(1.65-\alpha) / \sigma}$. When fitting the potential, We replace the Euclidean distance on the lattice by an improved distance $r_{I} / a$ which relates the separation between the static quark and anti-quark sources to the Fourier transform of the tree-level lattice gluon propagator. This procedure removes most of the short distance lattice artifacts. We show an example for a fit of the static quark potential with improved distances in Fig. 3 (left).

We have determined the scale parameter $r_{0}$ in units of the lattice spacing for 9 different parameter sets $\left(\hat{m}_{l}, \hat{m}_{s}, \beta\right)$. This allows to interpolate between different values of the gauge coupling and quark masses. We use a renormalization group inspired ansatz [20] which takes into account the quark mass dependence of $r_{0} / a$ [3] and which approaches, in the weak coupling limit, the 2-loop $\beta$-function for three massless flavors,

$$
\left(r_{0} / a\right)^{-1}=R(\beta)\left(1+B \hat{a}^{2}(\beta)+C \hat{a}^{4}(\beta)\right) \mathrm{e}^{A\left(2 \hat{m}_{l}+\hat{m}_{s}\right)+D} .
$$

Here $R(\beta)$ denotes the 2-loop $\beta$-function and $\hat{a}(\beta)=R(\beta) / R(\bar{\beta})$ with $\bar{\beta}=3.4$ chosen as an arbitrary normalization point. We use this interpolation formula to set the scale for the transition temperature, a fit is shown in Fig. 3 (right). 

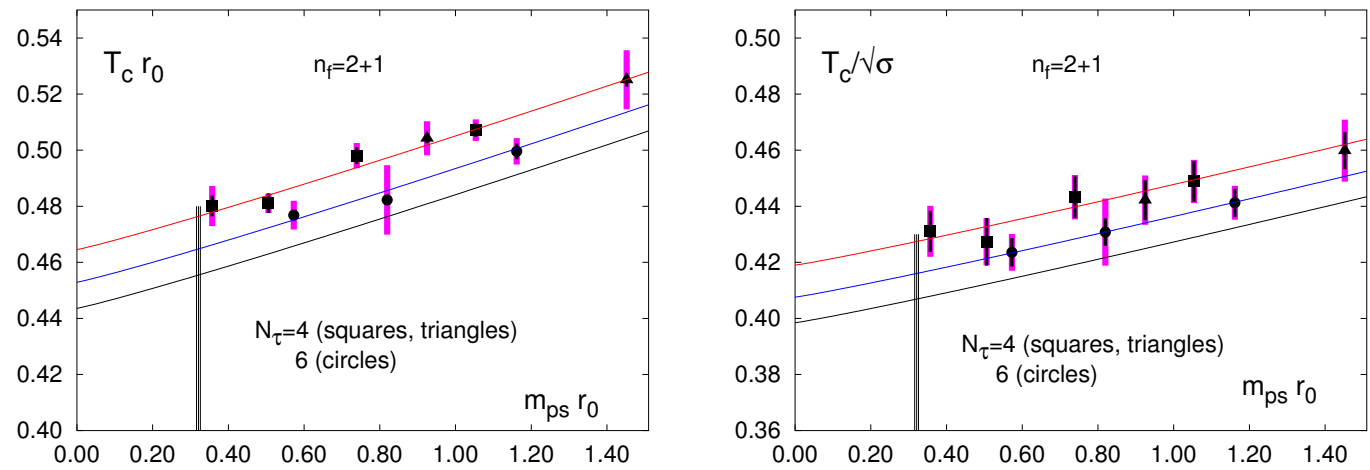

Figure 4: $T_{c} r_{0}$ (left) and $T_{c} / \sqrt{\sigma}$ (right) as a function of $m_{p s} r_{0}$ on lattices with temporal extent $N_{\tau}=4$, $\hat{m}_{s}=0.065$ (squares) and $\hat{m}_{s}=0.1$ (triangles) as well as for $N_{\tau}=6, \hat{m}_{s}=0.04$ (circles). Thin error bars represent the statistical and systematic error on $r_{0} / a$ and $\sqrt{\sigma} a$. The broad error bar combines this error with the error on $\beta_{c}$. The vertical line shows the location of the physical value $m_{p s} r_{0}=0.321(5)$ and its width represents the error on $r_{0}$. The three parallel lines show results of fits based on Eq. 5.1 with $d=1.08$ for $N_{\tau}=4,6$ and $N_{\tau} \rightarrow \infty$ (top to bottom).

\section{The transition temperature in $(2+1)$-flavor QCD}

To obtain the transition temperature we use the results for the scales $r_{0} / a$ and $\sqrt{\sigma} a$ obtained from fits to the static quark potential. In cases where zero temperature calculations have not been performed directly at the critical coupling but at a nearby $\beta$-value we use Eq. 4.3 to determine the scales at $\beta_{c}\left(\hat{m}_{l}, \hat{m}_{s}, N_{\tau}\right)$. The transition temperature is then obtained as $T_{c} r_{0} \equiv\left(r_{0} / a\right) / N_{\tau}$ or $T_{c} / \sqrt{\sigma}=1 / \sqrt{\sigma} a N_{\tau}$. We show these results as function of the pseudo-scalar (pion) mass expressed in units of $r_{0}$ in Figure 4. There we give 2 errors on $T_{c} r_{0}$ and $T_{c} / \sqrt{\sigma}$. A thin error bar reflects the combined statistical and systematic errors on the scales $r_{0} / a$ and $\sqrt{\sigma} a$ obtained from our 3parameter fit to the static quark potential. The broad error bar combines this uncertainty of the zero temperature scale determination with the scale-uncertainty arising from the error on $\beta_{c}$. As can be seen, the former error, which typically is of the order of $2 \%$, dominates our uncertainty on $T_{c} r_{0}$ and $T_{c} / \sqrt{\sigma}$ on the coarser $N_{\tau}=4$ lattices, while the uncertainty in the determination of $\beta_{c}$ becomes more relevant for $N_{\tau}=6$.

We have extrapolated our numerical results for $T_{c} r_{0}$ and $T_{c} / \sqrt{\sigma}$, which have been obtained for a specific set of lattice parameters $\left(\hat{m}_{l}, \hat{m}_{s}, N_{\tau}\right)$, to the chiral and continuum limit using an ansatz that takes into account the quadratic cut-off dependence, $(a T)^{2}=1 / N_{\tau}^{2}$, and a quark mass dependence expressed in terms of the pseudo-scalar meson mass,

$$
Y_{\hat{m}_{l}, \hat{m}_{s}, N_{\tau}}=Y_{0, m_{s}, \infty}+A\left(m_{p s} r_{0}\right)^{d}+B / N_{\tau}^{2} \quad, \quad Y=T_{c} r_{0}, T_{c} / \sqrt{\sigma},
$$

If the QCD transition is second order in the chiral limit the transition temperature is expected to depend on the quark mass as $\hat{m}_{l}^{1 / \beta \delta}$, or correspondingly on the pseudo-scalar meson mass as $m_{p s}^{2 / \beta \delta}$ with $d \equiv 2 / \beta \delta \simeq 1.08$ characterizing universal scaling behavior in the vicinity of second order phase transitions belonging to the universality class of $O(4)$ symmetric, 3-dimensional spin models. If, however, the transition becomes first order for small quark masses, which is not ruled 
out for physical values of the strange quark mass, the transition temperature will depend linearly on the quark mass $(d=2)$. A fit to our data set with $d$ as a free fit parameter would actually favor a value smaller than unity, although the error on $d$ is large in this case, $d=0.6(7)$.

Fortunately, the extrapolation to the physical point is not very sensitive to the choice of $d$ as our calculations have been performed close to this point. It does, however, increase the uncertainty on the extrapolation to the chiral limit. We have performed extrapolations to the chiral limit with $d$ varying between $d=1$ and $d=2$. From this we find

$$
m_{p s} r_{0} \equiv 0: \quad T_{c} r_{0}=0.444(6)_{-3}^{+12} \quad, \quad T_{c} / \sqrt{\sigma}=0.398(6)_{-1}^{+10},
$$

where the central value is given for fits with the $O(4)$ exponent $d=1.08$ and the lower and upper systematic error correspond to $d=1$ and $d=2$, respectively. Using the fit values for the parameter $A$ that controls the quark mass dependence of $T_{c} r_{0}(A=0.041(5))$ and $T_{c} / \sqrt{\sigma}(A=0.029(4))$, respectively, we can determine the transition temperature at the physical point, fixed by $m_{p s} r_{0}$ r, where we then obtain a slightly larger value with reduced systematic errors,

$$
m_{p s} r_{0} \equiv 0.321(5): \quad T_{c} r_{0}=0.457(7)_{-2}^{+8} \quad, \quad T_{c} / \sqrt{\sigma}=0.408(8)_{-1}^{+3}
$$

Here the error includes the uncertainty in the value for the physical point, $m_{p s} r_{0}$, arising from the uncertainty in the scale parameter $r_{0}=0.469(7) \mathrm{fm}$. We note that the extrapolated values for $T_{c} r_{0}$ and $T_{c} / \sqrt{\sigma}$ may also be interpreted as a continuum extrapolation of the shape parameters of the static potential. This yields $r_{0} \sqrt{\sigma} \simeq 1.11$ which is consistent with the continuum extrapolation obtained with the asqtad-action [3].

The fit parameter $B$ which controls the size of the cut-off dependent term in Eq. 5.1 is in all cases close to $1 / 3$. We find $B=0.34(9)$ for fits to $T_{c} r_{0}$ and $B=0.33(7)$ for fits to $T_{c} / \sqrt{\sigma}$, respectively. The critical temperatures for $N_{\tau}=4$ thus are about $5 \%$ larger than the extrapolated value, and for $N_{\tau}=6$ the difference is about $2 \%$. We therefore expect that any remaining uncertainties in our extrapolation to the continuum limit which may arise from higher order corrections in the cut-off dependence of $T_{c} r_{0}$ are not larger than $2 \%$.

The results for the transition temperature obtained here for smaller quark masses and smaller lattice spacings is entirely consistent with the results for 2-flavor QCD obtained previously with the p4fat 3 action on $N_{\tau}=4$ lattices in the chiral limit, $T_{c} / \sqrt{\sigma}=0.425(15)$ [11]. We now find for (2+1)flavor QCD for $N_{\tau}=4$ in the chiral limit $T_{c} / \sqrt{\sigma}=0.419(6)$. The continuum extrapolated result is, however, somewhat larger than the continuum extrapolated result obtained with the asqtad-action for (2+1)-flavor QCD in the chiral limit ${ }^{1}, T_{c} r_{0}=0.402$ (29) [3], which is based on the determination of transition temperatures on lattices with temporal extent $N_{\tau}=4,6$ and 8 .

Although we frequently have referred to the physical value of $r_{0}$ during the discussion in the previous chapters we stress that our final result for dimensionless quantities, in particular $T_{c} r_{0}$ and $T_{c} / \sqrt{\sigma}$ given in Eq. 5.2, does not depend on the actual physical value of $r_{0}$ or $\sqrt{\sigma}$.

At present the scale parameter $r_{0}$, deduced from the bottomonium level splitting using NRQCD [19], seems to be the best controlled lattice observable that can be used to set the scale for $T_{c}$. Using

\footnotetext{
${ }^{1}$ In [3] $T_{C}$ is given in units of $r_{1}$ using results for $r_{1} / a$ taken from [18]. We have expressed $T_{C}$ in units of $r_{0}$ using $r_{0} / r_{1}=1.4795$ to convert $r_{1}$ to the $r_{0}$ scale used by us.
} 
for $r_{0}$ the value given in Eq. 4.2 we obtain for the transition temperature in QCD at the physical point,

$$
T_{c}=192(7)(4) \mathrm{MeV},
$$

where the statistical error includes the errors given in Eq. 5.3 as well as the uncertainty in the value of $r_{0}$ and the second error reflects our estimate of a remaining systematic error on the extrapolation to the continuum limit. As discussed after Eq. 5.3 we estimate this error which arises from neglecting higher order cut-off effects in our ansatz for the continuum extrapolation, Eq. 5.1 to be about $2 \%$. Our resut for $T_{c}$ agrees within our current uncertainties with the result by the MILC Collaboration which is $T_{c}=169(12)(4)$ [3]. However, we encounter a discrepancy with the result by the Wuppertal group of $T_{c}=151(3)(3)$ [16] as determined by the chiral condensate.

\section{The equation of state}

We calculate the equation of state on a line of constant physics (LCP). Along this line the physical quark masses are fixed but the bare light and strange quark masses, which are the parameters in our action, change as a function of the coupling. We define the LCP by demanding that the ratio of strange pseudo-scalar mass over kaon mass $\left(m_{\bar{s} s} / m_{K}\right)$ as well as $m_{\bar{s} s}$ in units of the scale parameter $\left(r_{0} m_{\bar{s} s}\right)$ stay constant. We find that in good approximation the first condition is fulfilled by holding the ratio of bare light and strange quark mass $\left(\hat{m}_{l} / \hat{m}_{s}\right)$ fixed. We calculate meson masses in a wide range of the parameter space and use a renormalization group inspired interpolation formula to carefully tune the light quark masses as a function of the coupling $\beta$ in order to satisfy the second condition.

For calculating the pressure and the interaction measure we employ the integral method. Since the logarithm of the partition function can not be calculated easily on the lattice, derivatives with respect to the bare parameters are calculated $\left(\partial \ln Z / \partial \beta, \partial \ln Z / \partial \hat{m}_{l}, \partial \ln Z / \partial \hat{m}_{s}\right)$ and integrated along the LCP. We find for the pressure

$$
\begin{aligned}
\left.\frac{p}{T^{4}}\right|_{\beta_{0}} ^{\beta}= & N_{\tau}^{4} \int_{\beta_{0}}^{\beta} d \beta^{\prime}\left[\frac{1}{N_{\sigma}^{3} N_{\tau}}\left(\left\langle S_{G}\right\rangle_{0}-\left\langle S_{G}\right\rangle_{T}\right)\right. \\
& -\left(2\left(\langle\bar{\psi} \psi\rangle_{l 0}-\langle\bar{\psi} \psi\rangle_{l T}\right)+\frac{\hat{m}_{s}}{\hat{m}_{l}}\left(\langle\bar{\psi} \psi\rangle_{s 0}-\langle\bar{\psi} \psi\rangle_{s T}\right)\right)\left(\frac{\partial \hat{m}_{l}}{\partial \beta^{\prime}}\right)_{\hat{m}_{s} / \hat{m}_{l}} \\
& \left.-\hat{m}_{l}\left(\langle\bar{\psi} \psi\rangle_{s 0}-\langle\bar{\psi} \psi\rangle_{s T}\right)\left(\frac{\partial \hat{m}_{s} / \hat{m}_{l}}{\partial \beta^{\prime}}\right)_{\hat{m}_{l}}\right]
\end{aligned}
$$

As we find that $\hat{m}_{s} / \hat{m}_{l}=10$ can be kept fixed on a LCP, the last term in the integral vanishes. To calculate the interaction measure $\varepsilon-3 p$, the lattice $\beta$-function has to be known. Here we have

$$
\begin{aligned}
\frac{\varepsilon-3 p}{T^{4}} & =T \frac{\mathrm{d}}{\mathrm{d} T}\left(\frac{p}{T^{4}}\right)=-\left(a \frac{\mathrm{d} \beta}{\mathrm{d} a}\right)_{\mathrm{LCP}} \frac{\mathrm{d} p / T^{4}}{\mathrm{~d} \beta} \\
& =\left(\frac{\varepsilon-3 p}{T^{4}}\right)_{\text {gluon }}+\left(\frac{\varepsilon-3 p}{T^{4}}\right)_{\text {fermion }}+\left(\frac{\varepsilon-3 p}{T^{4}}\right)_{\hat{m}_{s} / \hat{m}_{l}} .
\end{aligned}
$$

Again the last term vanishes to good accuracy on the LCP. We emphasize that a precise knowledge of the $\beta$-function, $a \mathrm{~d} \beta / \mathrm{d} a$, along the LCP is necessary to calculate the interaction measure and 

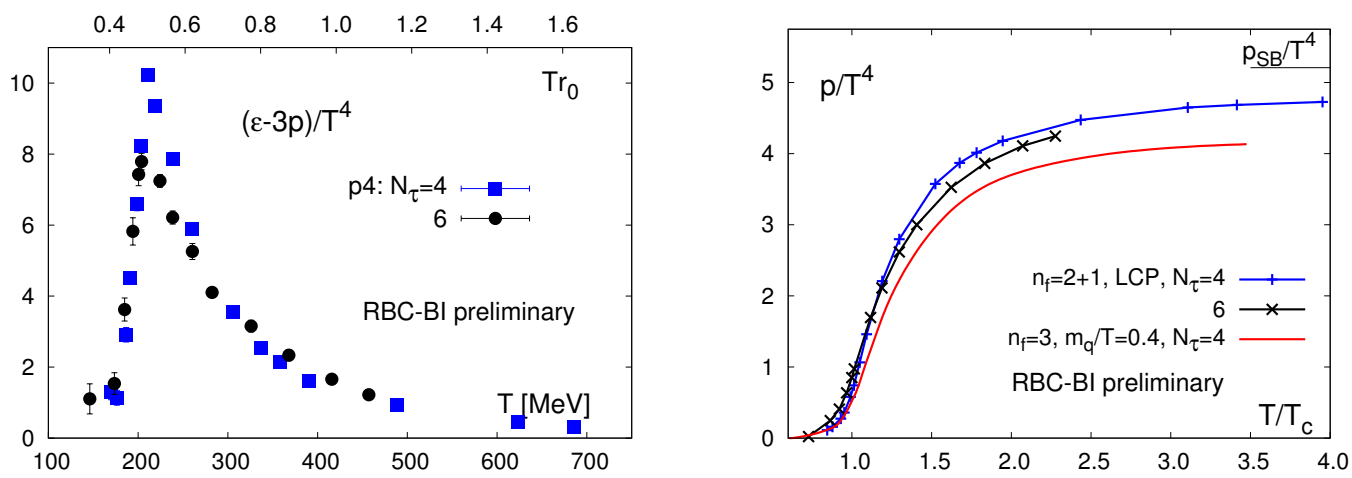

Figure 5: The interaction measure (left) and pressure (right) along a line of constant physics for $N_{\tau}=4$ and 6 . The ratio of light to strange quark mass has been held fixed at $\hat{m}_{l} / \hat{m}_{s}=0.1$. Also shown are earlier results for the pressure, obtained with 3-flavor QCD and const. $m_{q} / T=0.4$ [2].

energy density. We calculate the $\beta$-function from measurements of the scale parameter $r_{0}$, which we perform on each temperature (coupling) which we include in our equation of state.

In Fig. 5 we show our preliminary results for the interaction measure (left) and the pressure (right) on the LCP for $N_{\tau}=4$ and 6 . The ratio of light to strange quark mass has been held fixed at $\hat{m}_{l} / \hat{m}_{s}=0.1$. We compare our result for the pressure with earlier results for 3-flavor QCD and a constant $m_{q} / T=0.4$ [2]. One clearly sees a mass dependence, however, the mass dependence is small. Note that the quark masses differ by more than an order of magnitude in the high temperature region.

Or results on the interaction measure and pressure obtained with the p4fat 3 improved action are in complete agreement with corresponding results obtained with asqtad fermions [22]. Furthermore, the results show little cut-off dependence in the entire temperature regime analyzed. This is in contrast to calculations performed with the standard staggered discretization scheme [23], which leads to large cut-off effects in the hight temperature limit for lattices with temporal extent of $N_{\tau}=4$ and 6 .

\section{Hadronic fluctuations at zero and non-zero density}

It is conceptually very simple to calculate the expansion coefficients of any observable $O$ in a Taylor series around $\mu_{q}=0$ where $\mu_{q}$ is the quark chemical potential:

$$
O(\hat{\mu})=c_{0}+c_{1} \hat{\mu}+\frac{1}{2} c_{2} \hat{\mu}^{2}+\cdots .
$$

Since on the lattice all quantities are given in units of the lattice spacing $(a)$, the expansion parameter is $\hat{\mu} \equiv a \mu_{q}=N_{\tau}^{-1}\left(\mu_{q} / T\right)$. This idea goes back to the first calculation of the quark number susceptibility [24]. The response of hadron masses [25] as well as the pressure and further bulk thermodynamic quantities [7, 26, 27, 28] have been studied by this method. The first two nontrivial coefficients in Eq. (7.1) are given by

$$
c_{1}=\left\langle\frac{\partial O}{\partial \hat{\mu}}\right\rangle+\left\langle O \frac{\partial \ln \operatorname{detD}}{\partial \hat{\mu}}\right\rangle
$$




$$
c_{2}=\left\langle\frac{\partial^{2} O}{\partial \hat{\mu}^{2}}\right\rangle+2\left\langle\frac{\partial O}{\partial \hat{\mu}} \frac{\partial \ln \operatorname{det} \mathrm{D}}{\partial \hat{\mu}}\right\rangle+\left\langle O \frac{\partial^{2} \ln \operatorname{det} \mathrm{D}}{\partial \hat{\mu}^{2}}\right\rangle-\langle O\rangle\left\langle\frac{\partial^{2} \ln \operatorname{det} \mathrm{D}}{\partial \hat{\mu}^{2}}\right\rangle .
$$

Besides derivatives of the observable itself, the calculation of derivatives of $\ln \operatorname{det} D$ with respect to $\hat{\mu}$ is required. The derivatives have to be taken at $\hat{\mu}_{0}=0$. Note that due to the particle-antiparticle symmetry of the partition function $\left(Z\left(\mu_{q}\right)=Z\left(-\mu_{q}\right)\right)$ all odd coefficients in Eq. (7.1) vanish identically. For the same reason we have $\langle\partial \ln \operatorname{det} \mathrm{D} / \partial \hat{\mu}\rangle=0$ at $\hat{\mu}=0$. We explicitly use this property in Eq. (7.2) to derive the expansion coefficients.

The advantages of this method are that expectations values only have to be evaluated at $\hat{\mu}=0$, i.e. calculations are not directly affected by the sign problem. Furthermore, all derivatives of the fermion determinant can be expressed in terms of traces by using the identity $\ln \operatorname{det} \mathrm{D}=\operatorname{Tr} \ln \mathrm{D}$. This enables the stochastic calculation of the expansion coefficients by the random noise method, which is much faster than a direct evaluation of the determinant. Moreover, the continuum and infinite volume extrapolations are well defined on a coefficient by coefficient basis.

Quark number fluctuations $\chi_{q}$ belong to the most important observables that allow to follow the transition line into the non-zero chemical potential plane. They diverge at the critical end-point and thus provide an excellent signal for its existence and location. Eventually these fluctuations may be detectable in heavy ion experiments. Hadronic fluctuations can be computed from Taylor expansion coefficients of the pressure with respect to the quark chemical potential:

$$
\frac{p}{T^{4}}=\sum_{n=0}^{\infty} c_{n}(T)\left(\frac{\mu_{q}}{T}\right)^{n} \quad \text { with } \quad c_{n}(T)=\left.\frac{1}{n !} \frac{N_{\tau}^{3}}{N_{s}^{3}} \frac{\partial \ln Z}{\partial\left(\hat{\mu} N_{\tau}\right)^{n}}\right|_{\hat{\mu}=0} .
$$

Note that in the Taylor expansion of the pressure the up and down quark chemical potentials have been chosen to be equal. Having calculated the coefficients $c_{n}(T)$ one can construct the quark number density and quark number fluctuations

$$
\frac{n_{q}}{T^{3}}=\sum_{n=2}^{\infty} n c_{n}(T)\left(\frac{\mu_{q}}{T}\right)^{n-1} \quad ; \quad \frac{\chi_{q}}{T^{2}}=\sum_{n=2}^{\infty} n(n-1) c_{n}(T)\left(\frac{\mu_{q}}{T}\right)^{n-2} .
$$

In the case of two flavors of p4-improved staggered fermions, with $m_{q} / T=0.4$ the first three non-zero coefficients $c_{2}, c_{4}$, and $c_{6}$ have been calculated [27] and are shown in Fig. 6. We also show our preliminary results on $c_{2}$ for (2+1)-flavor QCD and a quark mass ratio of $\hat{m}_{q} / \hat{m}_{s}=0.1$. A mass dependence is clearly evident.

In Fig. 7 (left) we show the quartic quark number fluctuations of strange quarks from our ongoing (2+1)-flavor QCD simulations. which are given by $d_{4}^{S} \equiv\left(1 /\left(V T^{3}\right)\right)\left(\partial^{2} \ln Z / \partial\left(\mu_{s} / T\right)^{2}\right)$. As one can see also the quartic strange quark fluctuations show a peak at $T_{c}$.

In Fig. 7(right) we show the quark number fluctuations for $n_{f}=2$ and $m_{q} / T=0.4$ [27] for several values of the quark chemical potential including only the leading $\left(m u_{q} / T\right)^{2}$ correction which is proportional to $c_{4}$. It is interesting to see that at $\mu_{q}=0, \chi_{q}$ shows a rapid but monotonic increase at the transition temperature, whereas a cusp is developing at $T_{c}\left(\mu_{q}\right)$ for $\mu_{q}>0$. This is a clear sign for approaching the critical end-point.

\section{The critical end-point}

Locating the critical point is one of the most challenging goals of lattice QCD calculations at finite chemical potential. The first attempt to locate the critical point used the reweighting method 

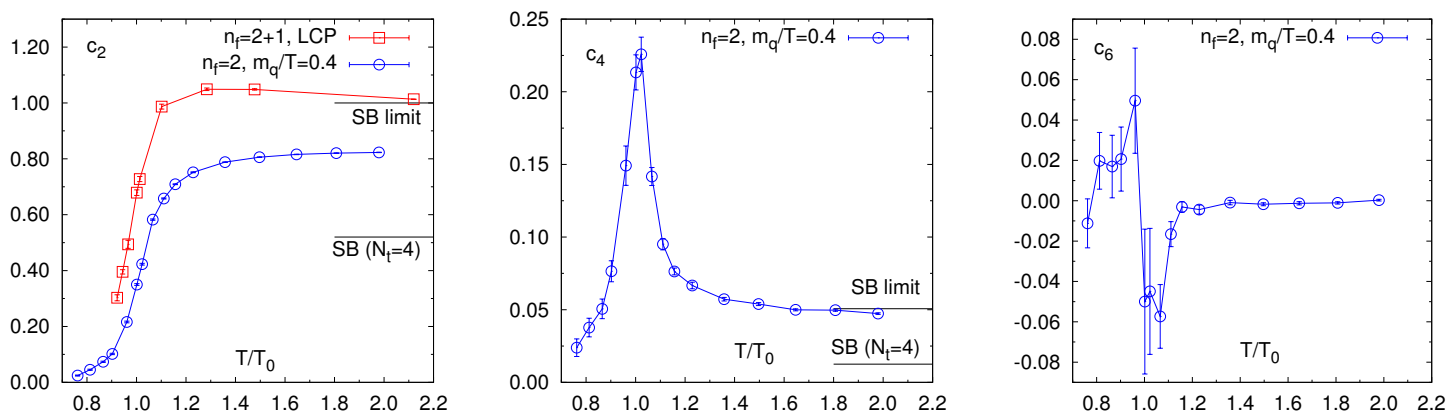

Figure 6: The Taylor expansion coefficients $c_{2}, c_{4}$ and $c_{6}$ of the pressure for $n_{f}=2$ and $m_{q} / T=0.4$ [27]. Also shown are preliminary results for (2+1)-flavor QCD and a quark mass ratio of $\hat{m}_{q} / \hat{m}_{s}=0.1$.
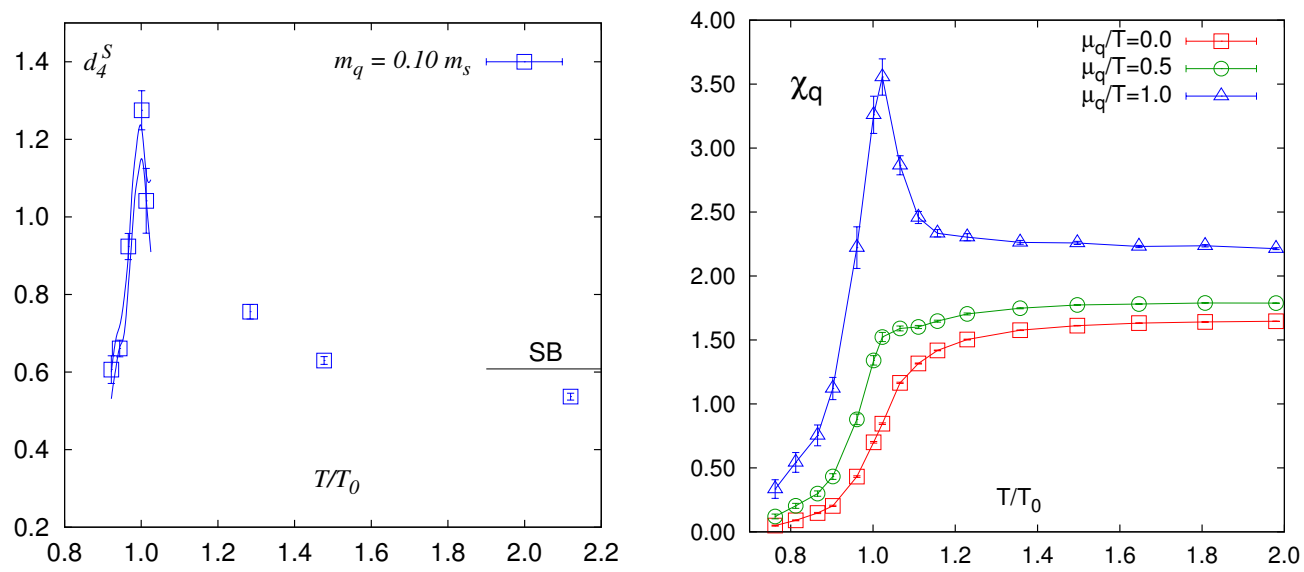

Figure 7: Quartic strange quark number fluctuations for (2+1)-flavor QCD and a quark mass ratio of $\hat{m}_{q} / \hat{m}_{s}=0.1$ (left) and the quark number susceptibility for $n_{f}=2$ and $m_{q} / T=0.4$ at several values of the quark chemical potential (right).

[29]. For this calculation, 2+1 flavor of standard staggered fermions have been used at a pion mass of about $300 \mathrm{MeV}$ and a kaon mass of about $500 \mathrm{MeV}$. Lattice sizes have, however, been rather small $\left(4^{3} \times 4-8^{3} \times 4\right)$. A critical chemical potential of $\mu_{B}^{c r i t}=725(35) \mathrm{MeV}$ was found. A second calculation [6], using again the reweighting method, with physical masses $\left(m_{\pi}=150 \mathrm{MeV}\right.$, $\left.m_{K}=500 \mathrm{MeV}\right)$ and somewhat larger volume $\left(6^{3} \times 4-12^{3} \times 4\right)$, let to $\mu_{B}^{\text {crit }}=360(4) \mathrm{MeV}$.

When using the reweighting method for locating the critical point, the minima of the normalized partition function in the complex $\beta$-plane (Lee-Yang zeros) have to be determined

$$
Z_{\text {norm }} \equiv\left|\frac{Z\left(\beta_{\operatorname{Re}}, \beta_{\operatorname{Im}}, \mu\right)}{Z\left(\beta_{\operatorname{Re}}, 0,0\right)}\right|=\left|\left\langle e^{6 i \beta N_{\tau} N_{\sigma}^{3} \Delta S_{G}} e^{i \theta} e^{\left(N_{f} / 4\right)(\operatorname{lndet} D(\mu)-\operatorname{lndet} D(0))}\right\rangle_{\left(\beta_{\mathrm{Re}, 0,0)}\right.}\right| .
$$

In $S U$ (3) gauge theory, where we have $e^{i \theta}=1$, this can be done with high accuracy [30]. In QCD with non-zero chemical potential the analysis of Lee-Yang zeros is, however, subtle [30]. For large volumes and chemical potentials the phase factor of the determinant $e^{i \theta}$ will force the Lee-Yang zero onto the real axis, which might lead to an underestimation of the critical point. 

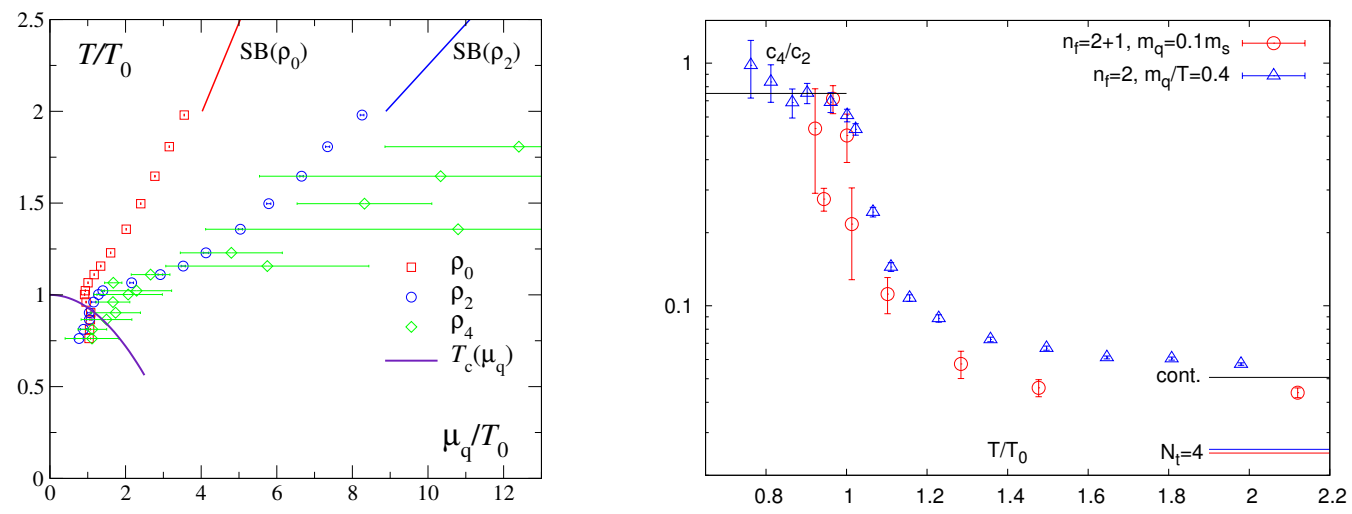

Figure 8: Estimates of the radius of convergence in the $\left(T, \mu_{q}\right)$-plane (left), the ratio $c_{4} / c_{2}$ of the expansion coefficients (right). The horizontal lines indicate the resonance gas limit $(T \rightarrow 0)$ and the SB limit in the continuum and at $N_{\tau}=4(T \rightarrow \infty)$.

Another difficulty with the reweighting method at finite chemical potential has been pointed out in [31]. It was noted, that taking the fourth (or square) root of the determinant (which is necessary in order to simulate 2 or 1-flavor QCD with staggered fermions; see also [32]) could lead to phase ambiguities. This problem becomes acute when $\mu_{q}>m_{\pi} / 2$.

All of the above mentioned limitations are, however, irrelevant for the location of the critical point with the reweighting method if the critical point is located at small values of $\mu_{q}$.

Using the Taylor expansion coefficients of the pressure, it is also possible to estimate the location of the critical point. The convergence radius of the expansion is limited by the nearest singularity in the complex chemical potential plane. For each fixed temperature, the radius of convergence is given by

$$
\rho=\lim _{n \rightarrow \infty} \rho_{n}=\lim _{n \rightarrow \infty} \sqrt{\left|\frac{c_{n}}{c_{n+2}}\right|} .
$$

Moreover, the sign of the coefficients $c_{n}$ gives information about the location of the singularity in the complex plane. If all coefficients are positive, the singularity is located on the real axis of the complex chemical potential plane. If the sign is strictly alternating, the singularity lies on the imaginary axis. For a detailed discussion see [33].

Having only a limited number of expansion coefficients, one can only estimate $\rho$. The hope is that the convergence of the $\rho_{n}$ will be fast. Indeed, a clustering of the $\rho_{n}$ is seen in the phase diagram, as shown in Fig. 8 [27]. This calculation, which has been performed with 2 flavors of $\mathrm{p} 4$ improved fermions and $m_{\pi} / m_{\rho}=0.7$, suggests a critical chemical potential of $\mu_{B}^{\text {crit }} \approx 500 \mathrm{MeV}$. All calculated $\rho_{n}$ are, however, consistent (within statistical error) with the resonance gas model in the Boltzmann approximation, where the radius of convergence is infinity.

The authors of [34] have estimated the critical chemical potential from a Taylor expansion of the quark number susceptibility and find $\mu_{B}^{c r i t} \approx 180 \mathrm{MeV}$. Two flavors of standard staggered fermions have been used on lattices up to $24^{3} \times 4$ and quark mass corresponding to $m_{\pi} / m_{\rho}=0.3$. The difference between the two estimates [27, 34] of the critical point is large. We note that the second estimate comes from the expansion coefficients of $\chi_{q}$. As can be seen from Eq. 7.4 this 


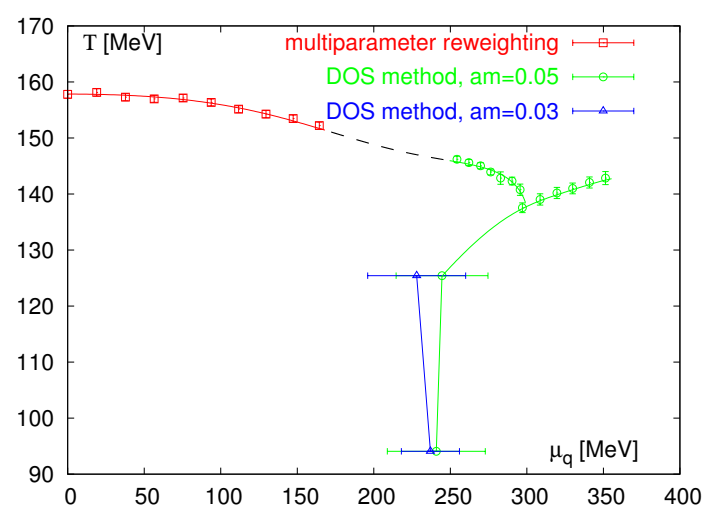

Figure 9: Phase diagram from the density of state method [35].

will result in a smaller $\rho_{n}$ for each fixed $n$. The $\operatorname{limit}_{\lim } \lim _{n \rightarrow \infty} \rho_{n}$ is of course the same. For finite $n$, however, the estimate of $\mu_{B}^{\text {crit }} \approx 180 \mathrm{MeV}$ would correspond to $\mu_{B}^{\text {crit }} \approx 240 \mathrm{MeV}$, when estimating the $\rho_{n}$ with coefficients of the same order from the expansion of the pressure. Nonetheless, the difference between the two estimates is still striking. The origin could be the difference in mass. However, preliminary results from the RBC-Bielefeld Collaboration, also shown in Fig. 8, do not indicate a strong mass dependence in $c_{4} / c_{2}=1 / \rho_{2}^{2}$.

\section{Beyond the critical point}

Even more challenging than locating the critical point, is the study of the physics at high densities and low temperatures. One attempt to do so is a calculation using the density of states method [35]. Using four flavors of standard staggered fermions (i.e. taking the root of the determinant is not necessary), several simulation points in the $(\beta, \hat{\mu})$ plane have been chosen to generate phase quenched configurations by employing the method proposed in [36]. The lattice size has been $6^{3} \times 4,6^{4}$ and $6^{3} \times 8$. The quark mass was chosen to be $m / T=0.3$. The generation has been done with constrained plaquettes. In oder to do so, we introduce a sharply peaked Gaussian potential in to the partition function, which in practice leads to a modification of the force term of the HMD-R algorithm. For each simulation point, several runs have been performed with about 20 different values of the plaquette. By calculating the eigenvalues of the reduced matrix the phase of the determinant was calculated for each of those runs. By numerically calculating the integrals

$$
\langle P\rangle=\int d x x \rho(x)\langle\cos (\theta)\rangle_{x} \quad\left\langle P^{2}\right\rangle=\int d x x^{2} \rho(x)\langle\cos (\theta)\rangle_{x},
$$

we recover the grand canonical expectation value of the plaquette and its square. Here $\rho(x)$ is the density of states, which has been measured by the integral method, usually used to calculate the pressure. The susceptibility of the plaquette is then given by the usual expression $\chi_{P}=\left\langle P^{2}\right\rangle-\langle P\rangle^{2}$. From the peak position of the plaquette susceptibility the phase diagram was calculated as shown in Fig. 9. The points at $T=93 \mathrm{MeV}$ are from calculations on $6^{3} \times 8$ lattices. Note, that we make no statement about the order of the transition lines. To determine the order of the transition one has to perform a finite-size-scaling analysis which is beyond the scope of this article. 
The plaquette expectation value and plaquette susceptibility suggest three different phases, which coincide in a triple point. The triple point is located around $\mu_{q}^{\mathrm{tri}} \approx 300 \mathrm{MeV}$, however its temperature $\left(T^{\text {tri }}\right)$ decreases from $T^{\text {tri }} \approx 148 \mathrm{MeV}$ on the $4^{4}$ lattice to $T^{\text {tri }} \approx 137 \mathrm{MeV}$ on the $6^{4}$ lattice. This shift reflects the relatively large cut-off effects one faces, with standard staggered fermions and temporal extents of 4 and 6.

The new phase at large chemical potentials and low temperatures is a natural candidate for a color superconducting phase. Recently, by combining experimental results from cold atoms in a trap [37] and some universal arguments, an upper bound for the transition line from the quark gluon plasma phase (QGP) to the superconducting phase (SC) was proposed $\left(T_{c} \leq 0.35 E_{F}\right)[38,39]$. To first approximation the Fermi-Energy $E_{F}$ is given by the chemical potential $\mu_{q}$. In [39] the triple point was estimated by comparing this upper bound with the experimental freeze-out curve. A temperature of $T^{\text {tri }} \leq 70 \mathrm{MeV}$ was found. Our value of the triple-point roughtly corresponds to $T_{c} \leq 0.46 E_{F}$. It is interesting that the two values are close.

At low temperatures we find a phase boundary which is very steep and almost independent of $\mu_{q}$. Although our lowest temperature is $96 \mathrm{MeV}$ an extrapolation to $T=0$ seems to be reasonable and would yield a critical chemical potential of $\mu_{q}(T=0) \approx 250 \mathrm{MeV}$ or equivalently $\mu_{B} / T_{c}\left(\mu_{B}=\right.$ $0) \approx 4$.7. This number appears to be at the lower edge of the phenomenological expectation of $\mu_{B} / T_{c}\left(\mu_{B}=0\right) \approx 5-10$. Note, that our lattice spacing is close to the strong coupling regime and we should feel the influence of the strong coupling limit. Strong coupling expansion calculations in general yield much lower values of $\mu_{B} / T_{c}\left(\mu_{B}=0\right) \lesssim 1.5$ [40].

For this work the density of state method has been employed, which works well on small lattices up to chemical potentials of $\mu_{q} / T \lesssim 3$ (other methods $[6,7,8]$ worked up to $\mu_{q} / T \lesssim 1$ ). The method is however extremely expensive and thus will in the near future not yield results close to the thermodynamic limit or the continuum limit, due to limitations in computer resources.

We have to emphasize that this simulations have been carried out on coarse lattices with an unphysical value of $n_{f}=4$ degenerate fermion flavor, and that neither the continuum nor the thermodynamic limit has been taken. Since we used unimproved staggered fermions, the corrections due to a finite lattice spacing are large. We also expect corrections due to the finite size of our volume. The simulations have not been performed with a constant quark mass, but $m_{q} / T=0.3$ has been held fixed.

\section{Acknowledgments}

I would like to thank F. Karsch and Z. Fodor for helpful discussions and comments. All members of the RBC-Bielefeld Collaboration are gratefully acknowledged for providing me with preliminary data. This work has been supported by the U.S. Department of Energy under contract DE-AC02-98CH1-886.

\section{References}

[1] F. Karsch, Lect. Notes Phys. 583, 209 (2002);

E. Laermann and O. Philipsen, Ann. Rev. Nucl. Part. Sci. 53, 163 (2003)

[2] F. Karsch, E. Laermann and A. Peikert, Phys. Lett. B 478, 447 (2000) 
[3] C. Bernard et al. [MILC Collaboration], Phys. Rev. D 71, 034504 (2005).

[4] C. Bernard et al., PoS LAT2005, 156 (2005).

[5] Y. Aoki, Z. Fodor, S. D. Katz and K. K. Szabo, JHEP 0601, 089 (2006).

[6] Z. Fodor and S. D. Katz, JHEP 0404 (2004) 050.

[7] R. V. Gavai and S. Gupta, Phys. Rev. D 68 (2003) 034506.

[8] Z. Fodor and S. D. Katz, Phys. Lett. B 534 (2002) 87; Z. Fodor and S. D. Katz, JHEP 0203 (2002) 014; C. R. Allton et al., Phys. Rev. D 66 (2002) 074507; P. R. Crompton, arXiv:hep-lat/0301001; M. D'Elia and M. P. Lombardo, Phys. Rev. D 67 (2003) 014505; M. D'Elia and M. P. Lombardo, Phys. Rev. D 70 (2004) 074509; Ph. de Forcrand and O. Philipsen, Nucl. Phys. B 642 (2002) 290; Ph. de Forcrand and O. Philipsen, Nucl. Phys. B 673 (2003) 170; V. Azcoiti, G. Di Carlo, A. Galante and V. Laliena, Nucl. Phys. B 723, (2005) 77.

[9] U. M. Heller, F. Karsch and B. Sturm, Phys. Rev. D 60, 114502 (1999).

[10] A. Peikert, B. Beinlich, A. Bicker, F. Karsch and E. Laermann, Nucl. Phys. Proc. Suppl. 63, 895 (1998); M. Cheng [RBC-Bielefeld Collaboration], PoS LAT2005, 045 (2006).

[11] F. Karsch, E. Laermann, A. Peikert, Nucl. Phys. B 605 (2001) 579.

[12] M. Cheng et al. [RBC-Bielefeld Collaboration], Phys. Rev. D 74 (2006) 054507.

[13] I. Horváth, A. D. Kennedy and S. Sint, Nucl. Phys. B 73, 834 (1999); M. A. Clark, A. D. Kennedy and Z. Sroczynski, Nucl. Phys. Proc. Suppl. 140, 835 (2005).

[14] S. Gottlieb, W. Liu, D. Toussaint, R. L. Renken and R. L. Sugar, Phys. Rev. D 35, 2531 (1987).

[15] RBC-Bielefeld Collaboration in preparation.

[16] Y. Aoki, Z. Fodor, S. D. Katz and K. K. Szabo, Phys. Lett. B 643 (2006) 46.

[17] R. Sommer, Nucl. Phys. B411 (1994) 839.

[18] C. Aubin et al. [MILC Collaboration], Phys. Rev. D70 (2004) 094505.

[19] A. Gray et al., Phys. Rev. D72 (2005) 094507.

[20] C. Allton, Nucl. Phys. B [Proc. Suppl.] 53, 867 (1997).

[21] M. Cheng et al., arXiv:hep-lat/0612001.

[22] C. Bernard et al., arXiv:hep-lat/0611031.

[23] Y. Aoki, Z. Fodor, S. D. Katz and K. K. Szabo, JHEP 0601 (2006) 089.

[24] S. A. Gottlieb et al., Phys. Rev. D 38 (1988) 2888.

[25] S. Choe et al. [QCD-TARO Collaboration], Nucl. Phys. Proc. Suppl. 106 (2002) 462; Phys. Rev. D 65 (2002) 054501; Nucl. Phys. A 698 (2002) 395.

[26] C. R. Allton et al., Phys. Rev. D 68 (2003) 014507.

[27] C. R. Allton et al., Phys. Rev. D 71 (2005) 054508.

[28] S. Ejiri, F. Karsch, E. Laermann and C. Schmidt, Phys. Rev. D 73 (2006) 054506.

[29] Z. Fodor and S. D. Katz, JHEP 0203 (2002) 014.

[30] S. Ejiri, Phys. Rev. D 73 (2006) 054502. 
[31] M. Golterman, Y. Shamir and B. Svetitsky, Phys. Rev. D 74 (2006) 071501; B. Svetitsky, Y. Shamir and M. Golterman, PoS LAT2006 148 (2006).

[32] S. R. Sharpe, PoS LAT2006 022 (2006).

[33] M. A. Stephanov, Phys. Rev. D 73 (2006) 094508; PoS LAT2006 024 (2006).

[34] R. V. Gavai and S. Gupta, Phys. Rev. D 71 (2005) 114014.

[35] C. Schmidt, Z. Fodor and S. D. Katz, arXiv:hep-lat/0512032; PoS LAT2005 (2006) 163.

[36] J. B. Kogut and D. K. Sinclair, Phys. Rev. D 66 (2002) 034505.

[37] V.S. Stepanyuk, L. Niebergall, W. Hergert, P. Bruno, arXiv:cond-mat/0502506.

[38] T. Schafer, arXiv:nucl-th/0606019.

[39] E. V. Shuryak, arXiv:nucl-th/0606046.

[40] N. Kawamoto, K. Miura, A. Ohnishi and T. Ohnuma, arXiv:hep-lat/0512023 and references therein. 